CIFRAS DE HOY

Rev Chil Salud Pública 2020,

Vol 24(2) 161- 165
Alberto Oyarzún-Serrano, Docente Internado APS, Escuela de Medicina, Universidad de Valparaíso, Campus San Felipe Médico Centro de Salud Familiar Valle los Libertadores, Putaendo. Tutor Internado APS

Paula Guevara-Zamora, Interna medicina VII año, Universidad de Valparaíso, Campus San Felipe

Camila Martínez-Silva, Interna medicina VII año, Universidad de Valparaíso, Campus San Felipe

Gustavo Espinoza-Olguín, Interno medicina VII año, Universidad de Valparaíso, Campus San Felipe

Oscar Valencia-Caicedo, Médico Centro de Salud Familiar Valle los Libertadores, Putaendo. Tutor Internado APS

Mario Parada-Lezcano, Docente Internado APS, Escuela de Medicina, Universidad de Valparaíso, Campus San Felipe

María José Cárdenas, Docente Internado APS, Escuela de Medicina, Universidad de Valparaíso, Campus San Felipe mariajose.cardenas@uv.cl

\section{CARACTERIZACIÓN DE PERSONAS CON ENFERMEDAD DE CHAGAS EN EL CENTRO DE SALUD FAMILIAR VALLE LOS LIBERTADORES Y ESTABLECIMIENTOS DEPENDIENTES EN PUTAENDO}

\author{
CHARACTERIZATION OF INDIVIDUALS WITH CHAGAS DISEASE \\ TREATED IN THE VALLE LOS LIBERTADORES FAMILY HEALTH \\ CENTER AND ASSOCIATED ESTABLISHMENTS IN PUTAENDO, \\ CHILE
}

\section{RESUMEN}

Introducción: La enfermedad de Chagas es una patología parasitaria crónica y sistémica; Chile tiene una incidencia de 11,6 por 100.000 habitantes, presentándose mayoritariamente en zonas rurales y precordilleranas. Putaendo es una comuna de la $\checkmark$ región ubicada en una zona endémica, donde se desconoce la realidad epidemiológica actual de la enfermedad. El objetivo del estudio es caracterizar a los pacientes con Chagas inscritos en el Centro de Salud Familiar (CESFAM) Valle Los Libertadores en Putaendo.

Material y Métodos: El estudio tuvo un diseño transversal. Se incluyeron todos los registros de pacientes con diagnóstico de enfermedad de Chagas hasta el 30 de junio de año 2019, excluyéndose a pacientes fallecidos. Los datos fueron obtenidos de una base de datos anonimizada de dicho centro.

Resultados: Se encontró una prevalencia de Chagas de 108 enfermos por 100.000 personas registradas vivas; 98 de sexo femenino y 56 masculino, con mayor frecuencia entre los 50-59 años. Respecto al Fondo Nacional de Salud (FONASA), los tramos $A$ y $B$ (las personas con menores ingresos) concentran la mayor cantidad de pacientes con Chagas (87\%).

Discusión: Si bien se pudo caracterizar a la enfermedad, siendo más prevalente en personas adultas, mujeres y de bajos recursos, se requieren más estudios para objetivar el real estado de salud de estos pacientes.

Palabras claves: Chagas, APS rural, epidemiología.

\section{ABSTRACT}

Introduction: Chagas disease is a chronic and systemic parasitic pathology; Chile has an incidence of 11.6 per 100,000 inhabitants, occurring mainly in rural and foothills areas. Putaendo is a municipality of the $V$ region, located in an endemic area, where the current epidemiological reality of the disease is unknown. The objective of the study is to characterize patients with Chagas enrolled in the Family Health Center (CESFAM) Valle Los Libertadores in Putaendo, Chile.

Material and Methods: The study was cross-sectional. All records of patients diagnosed with Chagas disease up to June 30, 2019 were included, excluding deceased patients. The data was obtained from the participating centers' anonymized database.

Results: The prevalence of Chagas disease in the center was 108 per 100,000, with 98 female and 56 male patients and the highest prevalence among the group from 50 to 59 years of age. With respect to the National Health Fund (FONASA), levels A and $B$ (lower income individuals) concentrate the largest number of Chagas patients $(87 \%)$.

Discussion: Although the disease could be characterized as being more prevalent in adults, women, and those with low resources, additional studies are needed to objectify the real state of health of these patients.

Key words: Chagas, rural PHC, epidemiology 


\section{INTRODUCCIÓN}

La enfermedad de Chagas, también conocida como tripanosomiasis americana, es una zoonosis parasitaria crónica y sistémica causada por el parásito Trypanosoma Cruzi, el cual a través del vector hematófago llamado triatoma infestans causa la enfermedad. Este último es conocido en Chile como vinchuca ${ }^{1}$. Habita principalmente en zonas rurales. Su principal mecanismo de trasmisión a los seres humanos es a través de las heces del Triatoma Infestans, que al entrar en contacto con la dermis por excoriaciones en la piel humana logran obtener acceso sistémico ${ }^{2}$. Existen otras formas de transmisión no vectoriales como son la vía transfusional, transplacentaria, alimentarias y accidentes laborales ${ }^{3}$.

En la fase aguda de la enfermedad se puede presentar cefalea, fiebre, falta de apetito, mialgias, vómitos, diarrea, hepatomegalia, esplenomegalia y chagoma (lesión de aspecto duro, con calor local, indolora y pequeña secundaria al contacto con el vector). La fase crónica inicia con un periodo asintomático de 10 a 30 años y posteriormente se agrega manifestaciones cardiacas y digestivos ${ }^{1}$.

Según cifras reportadas por la Organización Mundial de la Salud (OMS) en marzo 2020, en el mundo el número de casos ha caído de 24 millones en 1984 a 6-7 millones, concentrados principalmente en América Latina, generando 14.000 defunciones al año1,4. La enfermedad es endémica en 21 países distribuidos desde México hasta Argentina, con una incidencia anual de 30.000 casos vectoriales en la Región de las Américas y 9.000 recién nacidos infectados durante el embarazo ${ }^{5}$. En Chile, entre 1990 y 2008, se registraron tasas de incidencia de 2,95 por cien mil habitantes. En el año 2009 aumentó a 6,8 por cien mil habitantes y en el año 2011 a 11,6 por cien mil habitantes ${ }^{6}$. Lo anterior se explica por el tamizaje universal de todos los donantes de sangre y la implementación de la nueva circular de vigilancia en 2011. Por otro lado, las tasas de mortalidad se han mantenido estables durante los últimos 10 años, con cifras entre 0,3 y 0,4 por 100 mil habitantes. Se estima que la población considerada como positiva alcanza las 98.000 personas $^{3}$. El 98,2\% de los casos se concentra entre la región de Arica y Parinacota y la región de O'Higgins, presentándose mayoritariamente en zonas rurales y pre cordilleranas. La mayor parte de los casos se agrupa en el grupo etario de los 50-65 años. Esto se condice con la evolución natural de la enfermedad, y con que se interrumpió la transmisión vectorial. A nivel local, en el 2017, la V Región tiene una tasa de notificación de enfermedad de Chagas de 11,4 por 100 mil habitantes ${ }^{3,6}$.

Putaendo es una ciudad ubicada en la $V$ Región, específicamente en el Valle del Aconcagua, con una población de 16.754 hasta el año 2017 según reportes del Instituto Nacional de Estadísticas, de los cuales 8.358 corresponden a hombres y 8.390 a mujeres; su población adulta mayor es de 3.366 siendo un $20 \%$ de su población total. Posee un alto índice de ruralidad (el $72,8 \%$ reside en zona rural y el $27,2 \%$ en zona urbana7), generando vulnerabilidad que precipita al hacinamiento, limitación al acceso de servicios básicos, baja escolaridad, falta de oportunidades laborales y recursos económicos. Sumado a lo anterior su ubicación como comuna en zona endémica para enfermedad de Chagas, la sitúa en una posición relevante para el control de esta enfermedad y aportar a la salud pública del país.

Su Red de Salud está conformada por un Hospital (San Antonio de Putaendo), un Centro de Salud Familiar (Valle Los Libertadores), 4 postas rurales (Guzmanes, Piguchén, La Orilla y Quebrada Herrera) y un Centro Comunitario de Salud Familiar (CECOSF Las Coimas), siendo estos 5 establecimientos dependientes del CESFAM ${ }^{8}$. Entre el CESFAM y sus centros dependientes logran atender 15.112 personas. Siendo una zona endémica y una enfermedad de notificación obligatoria (Decreto Supremo N$^{\circ} 158$ ), se desconoce su estado actual en esta localidad ${ }^{3,6}$.

Por la importancia y el impacto epidemiológico que tiene esta enfermedad, así como por la ausencia de estudios previos, surge la necesidad de conocer más de las características de esta patología en esta comuna. El objetivo general del trabajo es realizar una caracterización de las personas con enfermedad de Chagas que se atienden en el CESFAM Valle de Los Libertadores, comuna de Putaendo.

\section{MATERIAL Y MÉTODOS}

Este estudio tiene un diseño metodológico: a) descriptivo b) observacional y c) transversal, ya que, respectivamente, a) no pretende probar una hipótesis de trabajo, b) los investigadores no manipulan las variables, y c) busca cuantificar y caracterizar a todos los pacientes con Chagas de Putaendo en un momento dado, sin realizarles un seguimiento. El Comité Institucional de Ética Científica de la Universidad de Valparaíso aprobó el proyecto al trabajarse sobre una base de datos anonimizada de usuarios 
inscritos en el CESFAM que no explicita datos sensibles de los pacientes (datos que sólo disponen la encargada de epidemiología del CESFAM y el jefe de estadísticas del CESFAM). La base de datos incluyó el desglose por establecimiento en el cual los pacientes estaban inscritos, ya sea al CESFAM VaIle Los Libertadores propiamente tal o a las postas o CECOSF dependientes de éste; además incluyó cantidad de pacientes infectados y su distribución por sexo, edad y tramo del Fondo Nacional de Salud (FONASA), además de nacionalidad y etnia, ya que todas estas variables serían estudiadas. Los criterios de inclusión son: todos aquellos registros de personas diagnosticadas con enfermedad de Chagas desde el 01 de octubre de 2012 hasta el 30 de junio de año 2019 que pertenezcan al Centro de Salud Familiar Valle Los Libertadores. Los criterios de exclusión son todos aquellos registros de personas con la enfermedad pero que estaban fallecidas al momento de estudio.
Se realizó un análisis descriptivo de los datos utilizando Microsoft ExcelTM para el estudio de las variables antes citadas, en que se registraron todos los pacientes con enfermedad de Chagas por única vez (no se registraron nuevamente a pacientes con 2 o más atenciones de salud), no se registró población extranjera y se omitieron los pacientes fallecidos en el conteo.

\section{RESULTADOS}

De un total de 14.263 personas registradas vivas en la base de datos anonimizada que se atienden en la red de salud pública de Putaendo, hasta el 30 de junio de 2019, hay un total de 154 pacientes con diagnóstico de enfermedad de Chagas, con una prevalencia de 108 enfermos por 100.000 personas registradas vivas. En la tabla $N^{\circ} 1$ se describen a los pacientes con enfermedad de Chagas considerados en este estudio.

Tabla $N^{\circ}$ 1: Caracterización de pacientes con enfermedad de Chagas en CESFAM Valle los Libertadores y establecimientos dependientes hasta el 30 junio de 2019

\begin{tabular}{|c|c|c|c|c|c|c|c|c|}
\hline \multirow[b]{2}{*}{ Establecimiento } & & \multirow[b]{2}{*}{$\mathrm{n}^{0}$} & \multicolumn{2}{|c|}{ Sexo } & \multicolumn{3}{|c|}{ Tramo FONASA } & \multirow[b]{2}{*}{$\mathrm{D}$} \\
\hline & & & Femenino & Masculino & A & B & $\mathrm{C}$ & \\
\hline CESFAM Valle Libertadores & & 151 & 96 & 55 & 43 & 89 & 7 & 12 \\
\hline Posta la Orilla & & 1 & 1 & 0 & 1 & 0 & 0 & 0 \\
\hline CECOSF Las Coimas & & 2 & 1 & 1 & 0 & 1 & 1 & 0 \\
\hline \multirow{2}{*}{ TOTAL } & $n^{0}$ & 154 & 98 & 56 & 44 & 90 & 8 & 12 \\
\hline & $\%$ & 100 & 63,6 & 36,4 & 28,6 & 58,4 & 5,2 & 7,8 \\
\hline
\end{tabular}

En la tabla $\mathrm{N}^{\circ} 1$ se observa que el CESFAM Valle Los Libertadores concentra la mayoría de los casos (151) por establecimiento, además se verifica que, en la previsión de salud, el tramo FONASA B concentra la mayor cantidad de pacientes; respecto al sexo, la patología es más prevalente en mujeres 
Gráfico $N^{\circ}{ }^{1}$ : Enfermedad de Chagas por sexo y edad en CESFAM Valle Los Libertadores y establecimientos dependientes hasta el 30 de junio de 2019.

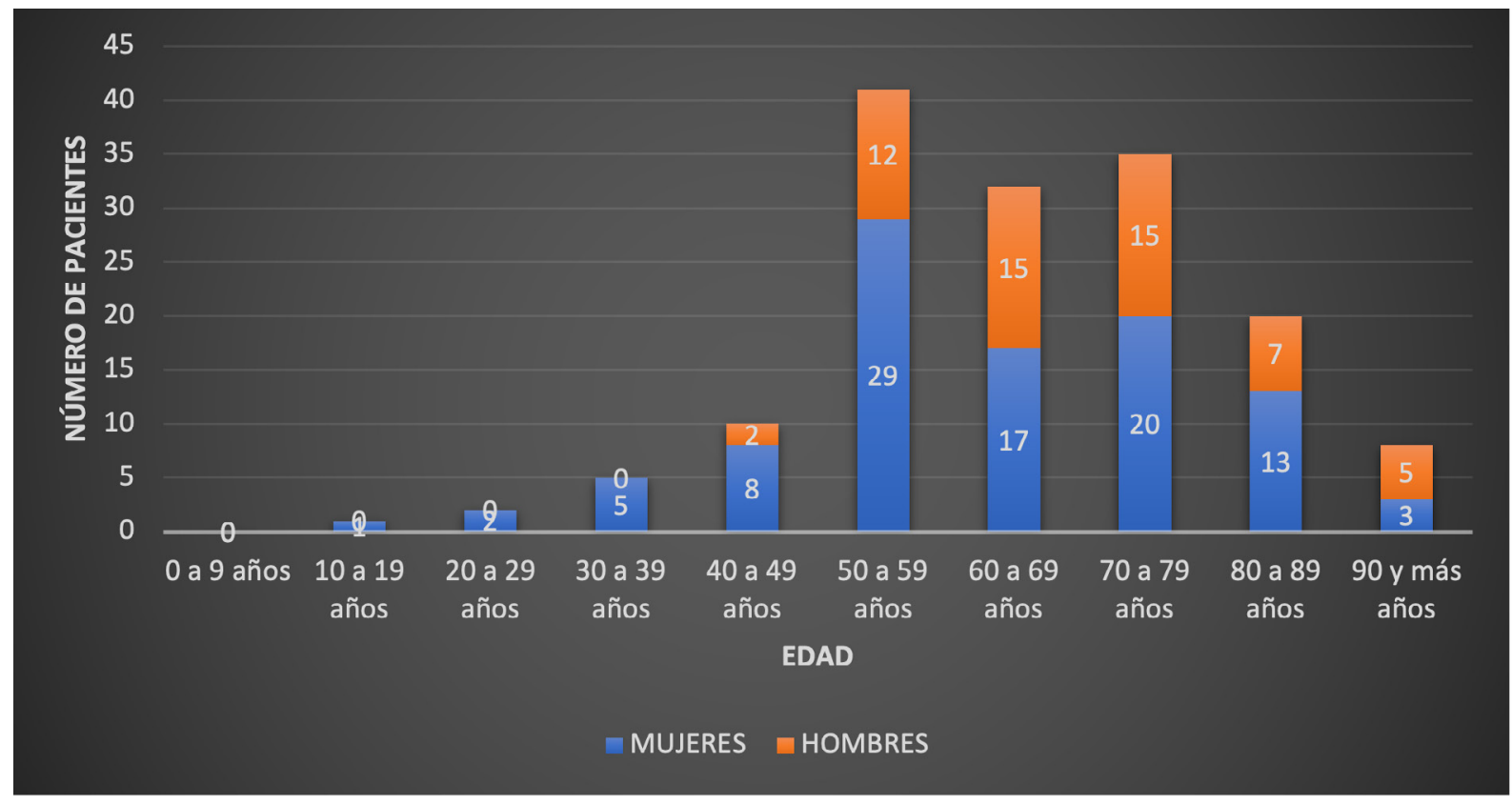

En el gráfico $\mathrm{N}^{\circ} 1$ se observa que la enfermedad es más frecuente en el grupo etario de 50-59 años y en las mujeres.

Por último, sólo 3 pacientes se identificaban con un pueblo originario, correspondiendo, en todos los casos, al mapuche.

\section{DISCUSIÓN}

Se constató que existen 154 pacientes con enfermedad de Chagas que se atienden en el CESFAM Valle Los Libertadores y sus establecimientos dependientes CECOSF Las Coimas y Posta la Orilla. En este estudio se observa una relación de 7 mujeres cada 4 hombres presentando la enfermedad, lo que se considera un hallazgo importante a mencionar ya que dicha proporción no se describe en la bibliografía revisada, aportando un nuevo antecedente. Se observó además que, a mayor edad, mayor prevalencia de la patología, encontrando la mayor frecuencia de enfermos a los 50-59 años, donde encontramos al $26,6 \%$ del total de pacientes. Luego de esta edad, la prevalencia comienza a descender. Esto se condice con la realidad nacional y también con la de otras regiones del país, donde para el año 2015 en la región metropolitana el $51 \%$ de los casos notificados eran de sexo femenino, y siendo el grupo de 50-59 años la generación con mayor número de casos positivos detectado ${ }^{9}$.

Respecto al tramo FONASA, la mayor parte de pacientes se ubica en el tramo B 58,4\%, seguido del tramo A $28,6 \%$. Esto significa que el $87 \%$ del total de enfermos tiene un ingreso mensual igual o menor a $\$ 250.000$. Lo anterior significa que es una patología más prevalente en grupos de escasos recursos socioeconómicos. No se encontraron datos comparativos a nivel nacional sobre el Chagas y la situación socioeconómica del paciente.

Una de las principales limitaciones de este estudio es que utiliza una base de datos secundaria en que los datos fueron recogidos para otro fin, por lo que la validez de tales datos pudiese ser cuestionada; en este mismo sentido, no se logró recabar información sobre el año de diagnóstico, complicaciones, tratamiento actual, seguimiento ni sobre otros determinantes de la salud implicados en la génesis de la enfermedad, con lo que se hubiese logrado caracterizar mejor a las personas que padecen de Chagas en Putaendo, ya que esta información se encuentra en otra base de datos hospitalaria a la cual no se tuvo acceso. Se deja la interrogante de estas variables epidemiológicas para continuar con el estudio en futuras investigaciones, para así terminar de de- 
finir la situación epidemiológica de la enfermedad de Chagas en una localidad endémica como lo es Putaendo.

Si bien es una enfermedad que ha disminuido su prevalencia en Putaendo ${ }^{5}$, a nivel nacional aún genera un $10 \%$ de enfermedades por dilatación del intestino grueso y esófago y un 30\% por dilatación e insuficiencia cardiaca ${ }^{9}$.

En el ámbito andino se han alcanzado importantes logros para cortar la transmisión, tales como: 1) la interrupción de la transmisión vectorial del Trypanosoma Cruzi por la Triatoma Infestans, 2) el establecimiento de una red de vigilancia y acciones de prevención en la región amazónica y 3) la atención a brotes de Chagas como enfermedad de transmisión alimentaria ${ }^{9}$. Conocer la realidad epidemiológica de la enfermedad en la Región, y sobre todo en localidades endémicas como lo es Putaendo, permitirá continuar y avanzar en todas estas estrategias de control de la enfermedad.

\section{REFERENCIAS BIBLIOGRÁFICAS}

1. Organizacion Mundial de la salud. Chagas disease (also known as American trypanosomiasis) [en línea]; 2020. [consultado en mayo de 2020]. Disponible en: https://www.who.int/news-room/fact-sheets/detail/ chagas-disease-(american-trypanosomiasis)

2. Malik LH, Singh GD, Amsterdam EA. Chagas Heart Disease: An Update. Am J Med. 2015 Nov;128(11):1251. e7-9.

3. Subsecretaría de Salud Pública. Circular de Vigencia de enfermedad de Chagas. Santiago, Chile; 2011.
4. Tasker R, Kliegman R, Nelson, Blum N, Shah S. Nelson. Tratado de Pediatría. 21st ed. Sciences EH; 2020.

5. Lorca M, Soto F, Soto P, Padilla G, Núñez E, Rojas J, Horta M, Bustamante M, Atencio J, Raychaudhuri S. Enfermedad de Chagas en las zonas rurales de la Región Metropolitana (Santiago) y de la V Región (Aconcagua). Rev Med Chil. 2008 Jul;136(7):945-6. .

6. Ministerio de Salud. Subsecretaría de Salud Pública Norma general técnica control y prevención nacional de la enfermedad de Chagas [en línea]; 2014. [consultado en junio de 2019]. Disponible en: https://www. minsal.cl/sites/default/files/NORMA\%20TECNICA_ CHAGAS_FINAL.pdf

7. Biblioteca del Congreso Nacional de Chile [en línea]. Santiago: BCN; [consultado en mayo de 2019]. Reportes estadísticos 2020. Disponible en: https://www. bcn.cl/siit/reportescomunales/comunas_v.html?anno $=2020$ \&idcom $=5705$.

8. Cesfam Valle los Libertadores [en línea]. Putaendo, Chile: CESFAM; [consultado en mayo de 2019]. Disponible en: http://www.cesfamputaendo.cl/?page=cesfam-home

9. Organismo Andino de salud - Convenio Hipólito Unanue. Plan estratégico de integración en salud 2018-2022 [en línea]. Lima: Tarea Asociación Gráfica Educativa; 2018 [consultado en junio de 2019]. Disponible en:http://www.orasconhu.org/portal/sites/ default/files/file/webfiles/doc/Plan_Estrategico_de_Integracion_en_Salud_2018_2022.pdf 